\title{
A 12-hour rapid titration method for cancer pain: a randomized, controlled, open-label study
}

\author{
Jianmiao Liang"^^, Lifu Chen", Shuang Yang, Hua Zhang, Limei Li, Zecheng Chen, Shunda Zhang, \\ Haibing Xian, Yicong Tang, Yanming Deng, Weineng Feng^ ${ }^{\wedge}$ \\ Department of Head and Neck/Thoracic Medical Oncology, The First People's Hospital of Foshan, Foshan, China \\ Contributions: (I) Conception and design: W Feng, J Liang, L Chen; (II) Administrative support: W Feng, Y Deng, L Chen; (III) Provision of study \\ materials or patients: J Liang, L Chen, S Yang, H Zhang, L Li, Z Chen, S Zhang, H Xian, Y Tang; (IV) Collection and assembly of data: J Liang, L \\ Chen; (V) Data analysis and interpretation: J Liang, W Feng; (VI) Manuscript writing: All authors; (VII) Final approval of manuscript: All authors. \\ \#These authors contributed equally to this work as first authors. \\ Correspondence to: Weineng Feng. Department of Head and Neck/Thoracic Medical Oncology, The First People's Hospital of Foshan, No.81, North \\ Lingnan Avenue, Chancheng District, Foshan 528041, China. Email: fengwn81@126.com.
}

Backgrounda Opioid titration is the best way to achieve a balance of pain relief and tolerable side effects for moderate-to-severe cancer pain. Rapid dose titration helps to achieve early analgesia. We explored the efficacy and safety of a 12-hour rapid dose titration in treating cancer pain.

Methods: Opioid-naïve patients with moderate-to-severe cancer pain were randomly divided into oxycodone group and morphine group. The medicines were adjusted to oxycodone sustained-release tablets after 12 hours, and the dose of oxycodone sustained-release tablets was adjusted every 12 hours. The analgesic efficacy and adverse reactions during the treatment were observed until the 72 nd hour.

Results: A total of 106 patients were included in the analysis, with 51 patients in the oxycodone group and 55 in the morphine group. The pain control rate of all patients reached $96.2 \% 24$ hours after treatment, and it was not significantly different between two groups $(\mathrm{P}=0.619)$. The proportion of Numeric Rating Scale (NRS) score that decreased by $\geq 50 \%$ was significantly higher in the oxycodone group than in the morphine group $(\mathrm{P}=0.013)$. In the first 12 hours and 24 hours, significantly lower proportions of patients in the oxycodone group experienced multiple episodes of breakthrough pain (BTP) than in the morphine group $(\mathrm{P}=0.032, \mathrm{P}=0.021$, respectively). The quality of life of the patients in the oxycodone group was significantly higher than that in the morphine group at the 24th hour $(\mathrm{P}=0.047)$, as was the degree to which the quality of life had improved $(\mathrm{P}<0.001)$. Only grade 1 or 2 adverse reactions were observed during the study period, and no significant difference between two groups.

Conclusions: The 12-hour rapid dose titration method can achieve early analgesia, with mild adverse reactions. In particular, the rapid titration method with background sustained-release oxycodone can reduce BTP episodes and achieve significant early pain relief.

Keywords: Cancer pain; titration; oxycodone sustained-release tablets; morphine immediate-release tablets; quality of life

Submitted Aug 31, 2020. Accepted for publication Jan 06, 2021.

doi: 10.21037/apm-20-2336

View this article at: http://dx.doi.org/10.21037/apm-20-2336

^ ORCID: Jianmiao Liang, 0000-0001-9982-8294; Weineng Feng, 0000-0001-5453-8605. 


\section{Introduction}

Cancer pain is one of the most common symptoms in cancer patients. It occurs throughout all stages (from diagnosis to palliative care) of cancer and affects the quality of life and survival of patients, especially in patients with advanced malignancies. One study found that the prevalence of cancer pain was as high as $64 \%$ in patients with advanced cancer, with $59 \%$ of patients experiencing pain during antitumor treatment, and $33 \%$ of patients still suffering from cancer pain after effective treatment (1). First presented in 1986, the World Health Organization (WHO) analgesic ladder has long been the guiding document for cancer pain management, which recommends opioids as a basic drug for treating moderate-to-severe cancer pain. However, the effective dosages of opioids for pain relief vary significantly from person to person. Opioid titration is the best way to achieve a balance of pain relief and tolerable side effects (2). According to the 2012 European Association for Palliative Care (EAPC) guidelines for the use of opioid analgesics to treat cancer pain, immediaterelease or sustained-release oral morphine and oxycodone can be used for opioid titration, and immediate-release opioids should be given as needed as a supplementary medicine to treat breakthrough pain (BTP) $(3,4)$. Clinically, cancer pain now needs to be controlled both effectively and quickly. In a prospective randomized controlled study, earlier palliative care (including effective cancer pain management) was associated with greater survival gain (5). During a standard dose titration process, the dosage of an opioid for the following day was adjusted according to the amount of drug consumption on the day every 24 hours to achieve stable pain control (SPC) (6). It was reported that the 24-hour response rate of the standard dose titration protocol ranged from $62 \%$ to $87.4 \%$ (7-9). Attempts have been made to shorten the dose adjustment to achieve quick analgesia. The results of a small-sample retrospective study suggested that dose adjustment every 12 hours was superior to dose adjustment every 24 hours in relieving pain (10). Unfortunately, few prospective studies have investigated this topic. In our current study, we prospectively applied a 12-hour dose adjustment protocol and assessed its safety and effectiveness; we further compared the safety and efficiency of sustained-release oxycodone and immediaterelease morphine in the protocol.

We present the following article in accordance with the CONSORT reporting checklist (available at http://dx.doi. org/10.21037/apm-20-2336).

\section{Methods}

\section{Study design}

This study was a prospective randomized, controlled, openlabel, non-inferiority trial that is registered in the Chinese Clinical Trial Registry (No. ChiCTR-TIR-17012925). It was approved by the Medical Ethics Committee of Foshan First People's Hospital (LSY2017 No. 5), and strictly followed the Declaration of Helsinki (as revised in 2013), with all the patients giving informed consent.

We began to enroll patients with moderate-to-severe cancer pain in our department after November 1, 2017. The inclusion criteria were as follows: (I) aged 18-75 years; (II) with pathologically or cytologically confirmed malignant tumors; (III) Numeric Rating Scale (NRS) $\geq 3$ (11); (IV) opioid-naïve; (V) no antitumor therapy received within 3 days before enrollment or during treatment; (VI) with an expected survival of $\geq 3$ months; (VII) with normal liver/ kidney functions; (VIII) without contraindications to opioid use; and (IX) without a history of alcohol or substance abuse.

The drugs used were OxyContin ${ }^{\circledR}$ (oxycodone hydrochloride sustained-release tablets) (Mundipharma, China) and morphine hydrochloride tablets (Shenyang First Pharmaceutical Co., Ltd., Northeast Pharmaceutical Group, China). The patients were 1:1 randomly divided into an oxycodone group or a morphine group using preset envelopes. The initial dose of morphine group was $10 \mathrm{mg}$ according to the NCCN guidelines (12), while the initial dose of oxycodone group was $10 \mathrm{mg}$ for moderate pain and $20 \mathrm{mg}$ for severe pain, respectively $(13,14)$, and titration in each patient lasted a total of 72 hours (Figure 1). The conversion ratio of oxycodone to morphine ranged from $2: 1$ to $1.5: 1$ (15). To reduce nighttime treatment, dose titration was initiated before noon for all patients.

\section{Main measures}

Pain was assessed using the NRS $(11,16)$. The primary endpoint was defined as the pain control rate (proportion of NRS $\leq 3$ ) at the 24th hour. The secondary endpoints included the proportion of the NRS score that had decreased by $\geq 30 \%$ (effective) or $\geq 50 \%$ (markedly effective) at the 24th hour and the proportions of BTP within the first 12 and 24 hours ( $\geq 2$ times within the first 12 hours or $\geq 3$ times within the first 24 hours). The patient's quality of life was evaluated by using the Edmonton Symptom Assessment System (ESAS) before treatment, and 24 and 

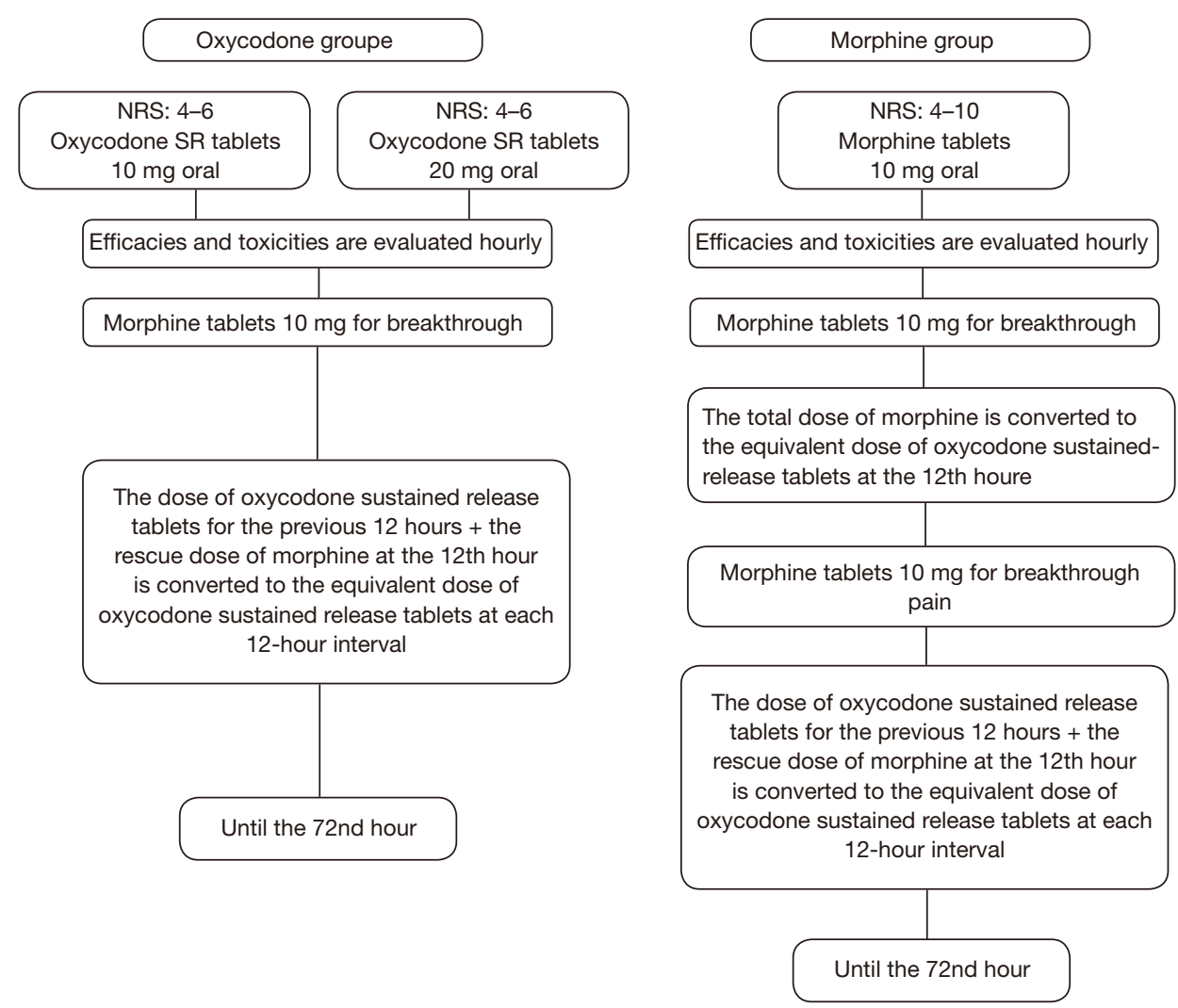

Figure 1 Titration algorithm of the two opioids. NRS, Numeric Rating Scale; Oxycodone SR, Oxycodone sustained-release.

72 hours after treatment (17). According to the Common Terminology Criteria for Adverse Events (CTCAE) 4.0, the adverse reactions were recorded during the treatment. All adverse reactions were treated according to European Society for Medical Oncology (ESMO) Clinical Practice Guidelines for management of cancer pain (4). The treatment should discontinue if severe adverse reactions occur or the patient requests to quit.

\section{Statistical analysis}

Based on the pain relief rates of immediate-release morphine titration and oxycodone hydrochloride sustainedrelease tablets titration in previous studies (7-9), the pain control rate was set to $80 \%$, with an $\alpha$ value of 0.05 and a $\beta$ value of 0.8 . The non-inferiority threshold was set to 0.20 . The calculated sample size was 100 patients; anticipating a $15 \%$ dropout rate, a total of 115 subjects were enrolled.

Statistical analysis was performed by using SPSS 19.0 software package (IBM, NY, USA). The measurement data are expressed as mean \pm standard deviations and were analyzed with $t$-test, descriptive analysis, and repeated measures analysis of variance. The intergroup comparisons of the count data were based on $\chi^{2}$ test. A value of $\mathrm{P}<0.05$ was considered statistically significant.

\section{Results}

\section{General information}

A total of 115 patients were enrolled from November 1, 2017 to June 14, 2019. After patients who violated the study protocol and those who asked to drop out during treatment were ruled out, 106 patients entered the final analysis, with 51 patients in the oxycodone group and 55 in the morphine group (Figure 2). The two groups were matched in terms of gender, age, smoking history, drinking history, disease type, and pain scores before the intervention (all $\mathrm{P}>0.05$ ) (Table 1).

\section{Analgesic efficacy}

\section{Pain control rate at the 24th hour}

The pain control rate at the 24th hour reached $96.2 \%$ (102 


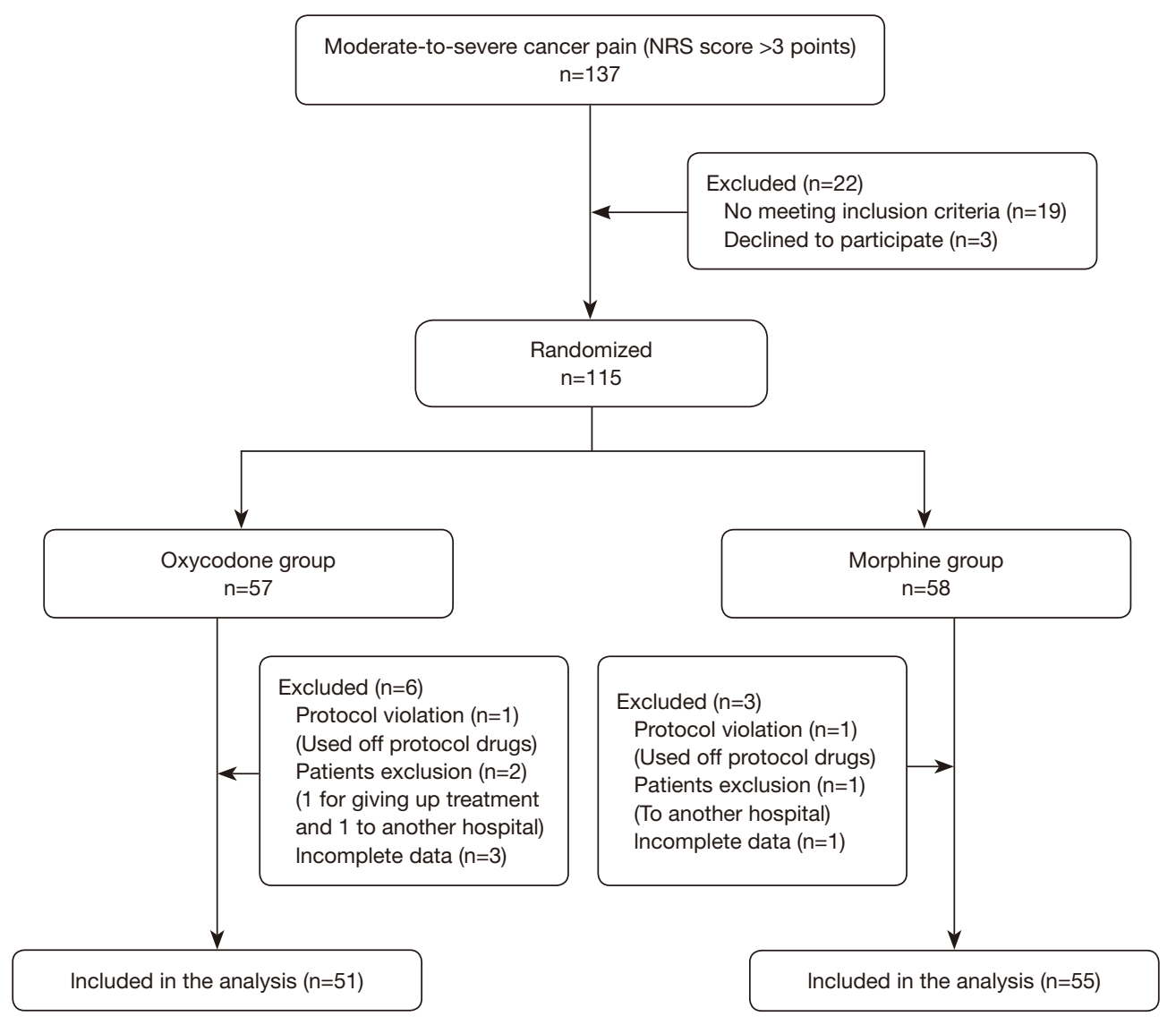

Figure 2 Patient enrollment algorithm. NRS, Numeric Rating Scale.

of all 106 patients); more specifically, it was $98.0 \%(50 / 51)$ in the oxycodone group and $94.5 \%(52 / 55)$ in the morphine group, showing no significant difference between these groups $(\mathrm{P}=0.619)$ (Figure 3). Evaluation of the average NRS scores at different time points showed that, although the overall NRS score was lower in the oxycodone group than in the morphine group, there were no significant differences at each time point (all $\mathrm{P}>0.05$ ) (Figure 4).

At the 24th hour, $88.7 \%$ of patients had an NRS score that had decreased by $\geq 30 \%$ (effective), and there was no significant difference between the oxycodone group and morphine group $(94.1 \%$ vs. $83.6 \%, \mathrm{P}=0.089) ; 80.2 \%$ of patients had a NRS score that had decreased by $\geq 50 \%$ (sufficiently effective), and the difference was statistically significant between these two groups $(90.2 \%$ vs. $70.9 \%$, $\mathrm{P}=0.013$ ) (Figure 5).

\section{BTP}

Within the first 12 hours of treatment, 9 (8.5\%) of 106 patients experienced two or more episodes of BTP; notably, significantly more patients in morphine group suffered from multiple BTP episodes ( $14.5 \%$ vs. $2 \%, \mathrm{P}=0.032)$. In contrast, $12(11.3 \%)$ of 106 patients experienced three or more episodes of BTP within the first 24 hours of treatment; again, the proportion of multiple BTP episodes ( $\geq 3$ times) was significantly higher in the morphine group than in the oxycodone group $(18.2 \%$ vs. $3.9 \%, \mathrm{P}=0.021)$ (Figure 6).

\section{Quality of life}

At the 24th hour, the ESAS score of the oxycodone group was significantly lower than that of the morphine group $(\mathrm{P}=0.047)$, and the decrease in the ESAS score was significantly larger in the oxycodone group than in the morphine group $(\mathrm{P}<0.001)$. At the $72 \mathrm{nd}$ hour, there was no significant difference in the ESAS score or its decline between these two groups $(\mathrm{P}=0.10, \mathrm{P}=0.527$, respectively) 
Table 1 Baseline patient characteristics

\begin{tabular}{|c|c|c|c|}
\hline Characteristics & Oxycodone group $(n=51)$ & Morphine group $(n=55)$ & $P$ value \\
\hline Male & $33(64.7 \%)$ & $38(69.1 \%)$ & \\
\hline Female & $18(35.3 \%)$ & $17(30.9 \%)$ & \\
\hline Age (years) & $59.11 \pm 10.11$ & $56.18 \pm 11.70$ & 0.298 \\
\hline Yes & $19(37.3 \%)$ & $20(36.4 \%)$ & \\
\hline No & $32(62.7 \%)$ & $35(63.6 \%)$ & \\
\hline Drinking & & & 0.457 \\
\hline Yes & $5(9.8 \%)$ & $8(14.5 \%)$ & \\
\hline Lung cancer & $40(78.4 \%)$ & $43(78.2 \%)$ & \\
\hline Head and neck cancer & $9(17.6 \%)$ & $8(14.5 \%)$ & \\
\hline Others & $2(3.9 \%)$ & $4(7.3 \%)$ & \\
\hline NRS & $4.39 \pm 0.6$ & $4.45 \pm 0.57$ & 0.901 \\
\hline ESAS & $24.18 \pm 7.27$ & $25.87 \pm 9.29$ & 0.211 \\
\hline
\end{tabular}

NRS, Numeric Rating Scale; ESAS, Edmonton Symptom Assessment System.

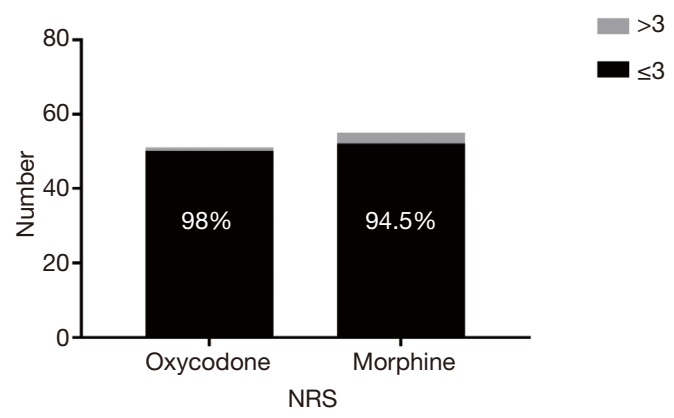

Figure 3 Proportions of NRS $\leq 3$ at the 24th hour. NRS, Numeric Rating Scale.

(Figure 7).

\section{Adverse reactions}

Forty patients [including 20 cases $(39.2 \%)$ in the oxycodone group and 20 cases $(36.4 \%)$ in the morphine group] experienced adverse reactions during the treatment. All the adverse reactions were grade 1 or 2 . No grade 3 or 4 adverse reactions were reported. Notably, up to $30 \%$ of

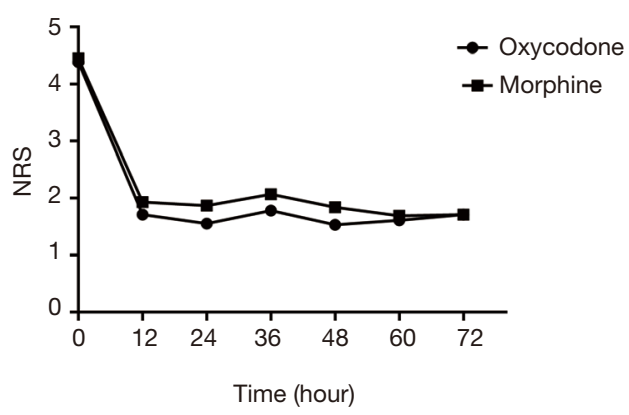

Figure 4 The NRS scores in the two groups during the treatment. NRS, Numeric Rating Scale.

patients experienced constipation. There was no significant difference in the incidence of adverse reactions between these two groups during the 72-hour treatment (Table 2).

\section{Discussion}

To the best of our knowledge, this was the first prospective study on opioid dose adjustment performed at 12-hour intervals. Furthermore, in order to obtain a faster and 


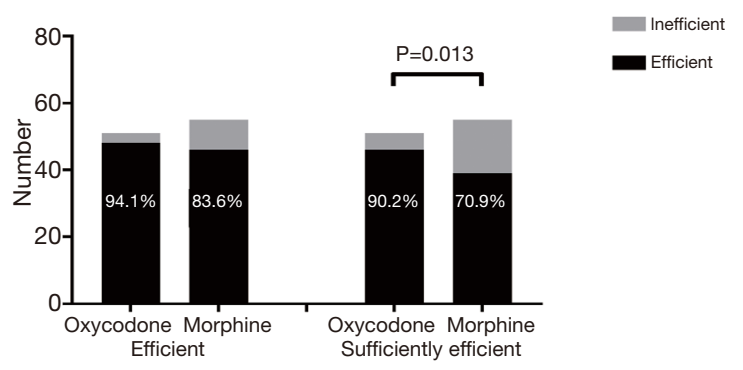

Figure 5 Proportions of NRS scores decreased by $\geq 30 \%$ (effective) or $\geq 50 \%$ (sufficiently effective) at the 24th hour in the two groups. NRS, Numeric Rating Scale.

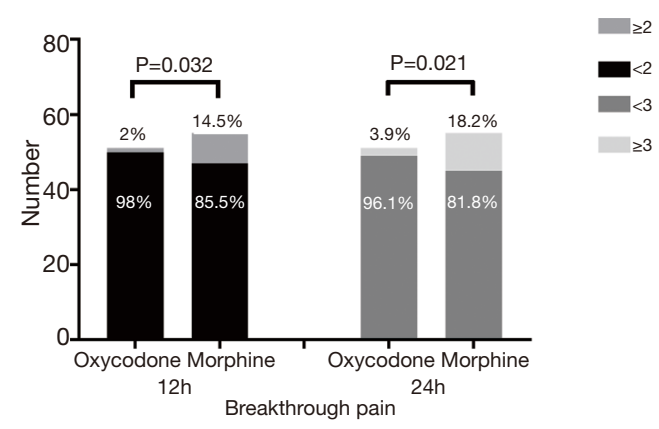

Figure 6 Proportions of multiple episodes of breakthrough pain within 12 and 24 hours.

better analgesic titration algorithm, the effect of oxycodone or morphine use in the first 12 hours of titration was examined, and these two methods were randomly compared. The 24 -hour pain control rate reached $96.2 \%$ in all patients. Both the oxycodone group and the morphine group had a pain control rate of over $90 \%(98.0 \%$ in the oxycodone group and $94.5 \%$ in the morphine group). It was reported that the 24-hour response rate of the standard dose titration protocol ranged between $62 \%$ and $87.4 \%$ (7-9). In a systematic review, Zhou et al. found the pain control rate of oxycodone and morphine to be $86 \%$ and $82.98 \%$, respectively (18). In our current study, the pain control rate at the 24th hour was significantly higher than that of previous standard titration protocols, showing that the 12-hour rapid dose titration algorithm has a good analgesic efficacy and can achieve the analgesic goal earlier.

Pain is highly subjective, and pain relief is currently evaluated by different scales (19). In our current study, the proportions of NRS scores that had decreased by $\geq 30 \%$ or $\geq 50 \%$ were used as a secondary evaluation indicator. The results showed that the proportion of patients with a
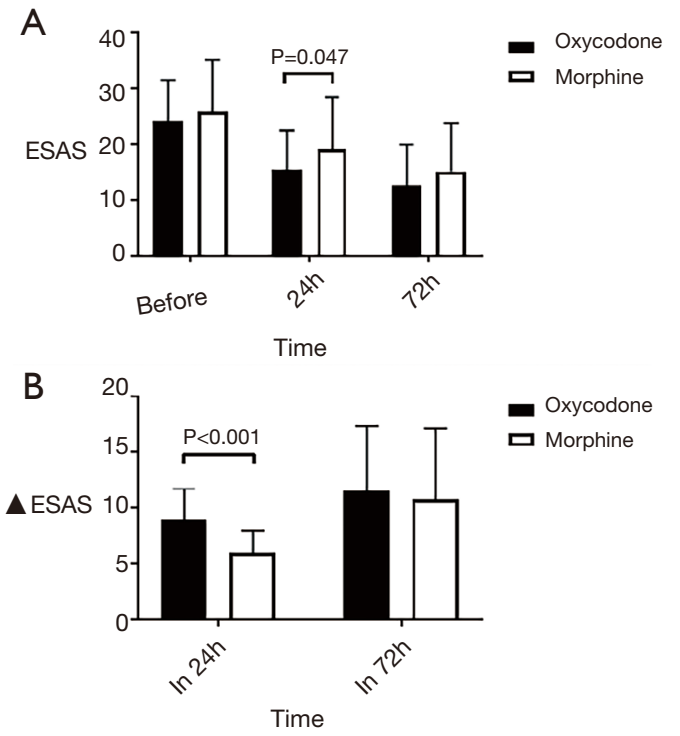

Figure 7 ESAS score and its decline ( $\mathbf{\Delta}$ ESAS). ESAS, Edmonton Symptom Assessment System.

NRS score that had decreased by $\geq 30 \%$ at the 24th hour reached $88.7 \%$, and the proportion of NRS scores that had decreased by $\geq 50 \%$ exceeded $80 \%$ in both the oxycodone group and morphine group. Lee et al. used the same method to evaluate the analgesic effects of oxycodone and morphine; on the first day, the proportion of NRS scores that had decreased by $\geq 30 \%$ and $\geq 50 \%$ was $54.4 \%$ and $36.4 \%$, respectively, in the oxycodone group and $46.9 \%$ and $25.0 \%$ in the morphine group (20). The proportions of NRS scores that had decreased by $\geq 30 \%$ and $\geq 50 \%$ were higher in our study than in Lee's study, suggesting the 12-hour rapid dose titration has a good analgesic effect.

Opioids block pain transduction to induce analgesia by stimulating opioid receptors. Opioids have different affinity and intrinsic activity towards different receptors. Different receptors produce different pharmacological effects after excitation. Morphine is a mu-opioid receptor agonist and weak kappa receptor agonist. Oxycodone is a semisynthetic morphine analogue that acts the mu-, delta-, and kappaopioid receptors and has 1.5 to 2 times the analgesic effect of oral morphine $(15,21)$. Previous studies have shown that the standard analgesic titration protocols using oxycodone or morphine showed no significant difference in analgesic effects $(9,22,23)$. However, another randomized controlled study showed that oxycodone sustained-release tablets had a superior time-to-analgesia onset compared to immediaterelease morphine, which helped to reduce titration 
Table 2 Analysis of adverse events

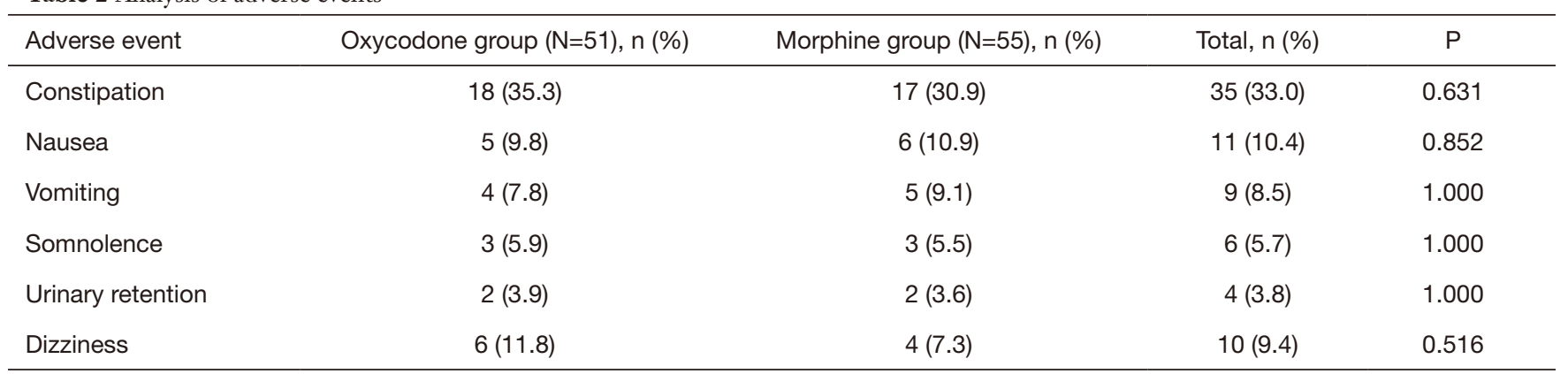

cycles (24). In our current study, when the 12-hour rapid titration was applied, the oxycodone group and morphine group had no significant difference in the pain control rate at the 24th hour, which was the same as what occurred with the standard titration protocol. However, the proportion of patients whose NRS score had decreased by $\geq 50 \%$ was significantly higher in the oxycodone group than in the morphine group (90.2\% vs. $70.9 \%, \mathrm{P}=0.013)$. The average NRS score of the oxycodone group was slightly lower than that of the morphine group. The difference in the average NRS score was relatively obvious at the beginning hours, but it was not statistically significant; furthermore, the NRS scores tended to become more similar as time passed. Our study also found that oxycodone sustained-release tablets reduced the number of BTP reports. The proportion of patients experiencing multiple BTP episodes within 12 and 24 hours in the oxycodone group was significantly lower than that in the morphine group ( $2 \%$ vs. $14.5 \%, \mathrm{P}=0.032 ; 3.9 \%$ vs. $18.2 \%, \mathrm{P}=0.021)$. Studies have demonstrated that cancer pain, if not treated effectively and timely, can cause central sensitization, which increases the sensitivity of the nervous system to pain, increase pain perception, and/or reduce the analgesic effect; as a result, patients are more likely to develop refractory pain. Early analgesia may increase the patient's pain threshold, thereby reducing the patient's pain perception and achieving better pain relief $(25,26)$. In our current study, the proportion of multiple BTP episodes was significantly lower in the oxycodone group than in the morphine group, which might be explained by the higher proportion of decreased NRS score in the oxycodone group.

Since early analgesia may increase the patient's pain threshold and thus reduce the patient's pain perception, it may achieve better pain relief and improve the patient's quality of life $(25,26)$. In our current study, the ESAS score, which reflects the quality of life, was significantly lower in the oxycodone group than in the morphine group at the 24th hour $(\mathrm{P}=0.047)$, and the decrease of ESAS score was significantly larger in the oxycodone group $(\mathrm{P}<0.001)$, suggesting the oxycodone group had higher quality of life than the morphine group. At the 72 nd hour, there was no significant difference in the ESAS score or its decline between these two groups $(\mathrm{P}=0.10, \mathrm{P}=0.527$, respectively), which further suggested that the quality of life of patients in the oxycodone group were improved in an earlier and more effective manner.

The main adverse reactions of opioids include constipation, nausea/vomiting, and dizziness, and the adverse reactions among different opioids do not vary markedly (27-29). The major adverse reaction in our current study was constipation (33.0\%), followed by nausea (10.4\%), dizziness $(9.4 \%)$, and vomiting $(8.5 \%)$. These adverse reactions were mild (grades 1 and 2), and no serious adverse reactions occurred. The constipation was relieved after using laxatives, and the treatment medications were not affected. There was no significant difference in adverse reactions between the oxycodone group and the morphine group.

However, our study had certain limitations. Due to the lack of random comparison with the standard 24-hour titration, our findings could not accurately reflect the advantages of the 12-hour dose titration method over the standard protocol.

\section{Conclusions}

Rapid analgesic titration with a 12-hour dose adjustment has a good and early analgesic efficacy with mild adverse reactions. The rapid titration method based on oxycodone sustained-release tablets can better lower NRS score, reduce BTP episodes, build patients' confidence in subsequent treatment, and improve the quality of life. Thus, the value represented by the 12-hour dose titration scheme warrants 
further investigation.

\section{Acknowledgments}

The authors wish to acknowledge J. Gray for English language editing. We would also like to acknowledge all the patients, staff, and researchers who contributed to this study.

Funding: None.

\section{Footnote}

Reporting Checklist: The authors have completed the CONSORT reporting checklist. Available at http://dx.doi. org/10.21037/apm-20-2336

Data Sharing Statement: Available at http://dx.doi. org/10.21037/apm-20-2336

Conflicts of Interest: All authors have completed the ICMJE uniform disclosure form (available at http://dx.doi. org/10.21037/apm-20-2336). The authors have no conflicts of interest to declare.

Ethical Statement: The authors are accountable for all aspects of the work in ensuring that questions related to the accuracy or integrity of any part of the work are appropriately investigated and resolved. It was approved by the Medical Ethics Committee of Foshan First People's Hospital (LSY2017 No. 5), and strictly followed the Declaration of Helsinki (as revised in 2013), with all the patients giving informed consent.

Open Access Statement: This is an Open Access article distributed in accordance with the Creative Commons Attribution-NonCommercial-NoDerivs 4.0 International License (CC BY-NC-ND 4.0), which permits the noncommercial replication and distribution of the article with the strict proviso that no changes or edits are made and the original work is properly cited (including links to both the formal publication through the relevant DOI and the license). See: https://creativecommons.org/licenses/by-nc-nd/4.0/.

\section{References}

1. van den Beuken-van Everdingen MH, de Rijke JM, Kessels AG, et al. Prevalence of pain in patients with cancer: a systematic review of the past 40 years. Ann Oncol
2007;18:1437-49.

2. Wong SS, Cheung CW. Optimization of opioid utility in cancer pain populations. Ann Palliat Med. 2020;9:558-70.

3. Caraceni A, Hanks G, Kaasa S, et al. Use of opioid analgesics in the treatment of cancer pain: evidencebased recommendations from the EAPC. Lancet Oncol 2012;13:e58-68.

4. Ripamonti CI, Santini D, Maranzano E, et al. Management of cancer pain: ESMO Clinical Practice Guidelines. Ann Oncol 2012;23 Suppl 7:vii139-54.

5. Zimmermann C, Swami N, Krzyzanowska M, et al. Early palliative care for patients with advanced cancer: a clusterrandomised controlled trial. Lancet 2014;383:1721-30.

6. Reis-Pina P, Sabri E, Birkett NJ, et al. Cancer-Related Pain: A Longitudinal Study of Time to Stable Pain Control and Its Clinicodemographic Predictors. J Pain Symptom Manage 2019;58:812-23.e2.

7. Samolsky Dekel BG, Tomasi M, Vasarri A, et al. Opioid titration with sustained-release oxycodone and immediaterelease morphine for moderate/severe cancer pain: a pilot assessment of the CoDem protocol. J Opioid Manag 2014;10:29-38.

8. Riley J, Branford R, Droney J, et al. Morphine or oxycodone for cancer-related pain? A randomized, open-label, controlled trial. J Pain Symptom Manage 2015;49:161-72.

9. Corli O, Floriani I, Roberto A, et al. Are strong opioids equally effective and safe in the treatment of chronic cancer pain? A multicenter randomized phase IV 'real life' trial on the variability of response to opioids. Ann Oncol 2016;27:1107-15.

10. Jun G, Jing L, Bo L, et al. Effect of hydrochloride oxycodone controlled-release tablets with dose titration for moderate or severe cancer pain. Chinese Journal of Cancer Prevention and Treatment 2017;24:1319-22.

11. Caraceni A, Cherny N, Fainsinger R, et al. Pain measurement tools and methods in clinical research in palliative care: recommendations of an Expert Working Group of the European Association of Palliative Care. J Pain Symptom Manage 2002;23:239-55.

12. National Comprehensive Cancer Network. NCCN clinical practice guidelines in Oncology: Adult Cancer Pain (Version2. 2017). Available online: http://www.ncen. org/professionals/physician_gls/pdf/pain.pdf. Accessed 2017-05-10.

13. Yu SY, OxyContin Tablets Postmarketing Surveillance Study Group C. Postmarketing surveillance study of OxyContin tablets for relieving moderate to severe cancer 
pain. Oncology 2008;74 Suppl 1:46-51.

14. Cancer pain expert group of Beijing pain treatment quality control and Improvement Center. Beijing Guidelines for Cancer Pain Management (2017). Chinese Journal of Pain Medicine 2017;23:881-9.

15. Nakamura A, Hasegawa $M$, Ito $H$, et al. Distinct relations among plasma concentrations required for different pharmacological effects in oxycodone, morphine, and fentanyl. J Pain Palliat Care Pharmacother 2011; 25:318-34.

16. Serlin RC, Mendoza TR, Nakamura Y, et al. When is cancer pain mild, moderate or severe? Grading pain severity by its interference with function. Pain 1995;61:277-84.

17. Bruera E, Kuehn N, Miller MJ, et al. The Edmonton Symptom Assessment System (ESAS): a simple method for the assessment of palliative care patients. J Palliat Care 1991;7:6-9.

18. Zhou J, Wang Y, Jiang G. Oxycodone versus morphine for cancer pain titration: A systematic review and pharmacoeconomic evaluation. PLoS One 2020;15:e0231763.

19. Farrar JT, Dworkin RH, Max MB. Use of the cumulative proportion of responders analysis graph to present pain data over a range of cut-off points: making clinical trial data more understandable. J Pain Symptom Manage 2006;31:369-77.

20. Lee KH, Kang JH, Oh HS, et al. Intravenous Oxycodone versus Intravenous Morphine in Cancer Pain: A Randomized, Open-Label, Parallel-Group, Active-Control Study. Pain Res Manag 2017;2017:9741729.

21. Nielsen CK, Ross FB, Lotfipour S, et al. Oxycodone and morphine have distinctly different pharmacological profiles: radioligand binding and behavioural studies in two rat models of neuropathic pain. Pain 2007;

Cite this article as: Liang $\mathrm{J}$, Chen L, Yang S, Zhang $\mathrm{H}, \mathrm{Li}$ L, Chen Z, Zhang S, Xian H, Tang Y, Deng Y, Feng W. A 12hour rapid titration method for cancer pain: a randomized, controlled, open-label study. Ann Palliat Med 2021;10(1):88-96. doi: 10.21037/apm-20-2336
132:289-300.

22. Guo KK, Deng CQ, Lu GJ, et al. Comparison of analgesic effect of oxycodone and morphine on patients with moderate and advanced cancer pain: a meta-analysis. BMC Anesthesiol 2018;18:132.

23. Lauretti GR, Oliveira GM, Pereira NL. Comparison of sustained-release morphine with sustained-release oxycodone in advanced cancer patients. Br J Cancer 2003;89:2027-30.

24. Pan H, Shen P, Shu Q, et al. Efficacy and safety of sustained-release oxycodone compared with immediaterelease morphine for pain titration in cancer patients: A multicenter, open-label, randomized controlled trial (SOCIAL). Medicine (Baltimore) 2019;98:e15505.

25. Bandieri E, Sichetti D, Romero M, et al. Impact of early access to a palliative/supportive care intervention on pain management in patients with cancer. Ann Oncol 2012;23:2016-20.

26. Tsuji H, Tetsunaga T, Tetsunaga $T$, et al. The factors driving self-efficacy in intractable chronic pain patients: a retrospective study. J Orthop Surg Res 2019;14:473.

27. Oosten AW, Oldenmenger WH, Mathijssen RH, et al. A Systematic Review of Prospective Studies Reporting Adverse Events of Commonly Used Opioids for CancerRelated Pain: A Call for the Use of Standardized Outcome Measures. J Pain 2015;16:935-46.

28. Ma H, Liu Y, Huang L, et al. The Adverse Events of Oxycodone in Cancer-Related Pain: A Systematic Review and Meta-Analysis of Randomized Controlled Trials. Medicine (Baltimore) 2016;95:e3341.

29. Schmidt-Hansen M, Bennett MI, Arnold S, et al. Efficacy, tolerability and acceptability of oxycodone for cancerrelated pain in adults: an updated Cochrane systematic review. BMJ Support Palliat Care 2018;8:117-28. 